\title{
Effects of Sodium Valproate, Levetiracetam and Phenytoin Therapy on Evoked Potentials in Children with Epilepsy
}

\author{
Jai Behgal ${ }^{1} \cdot$ Radhamohan Rana $^{1} \cdot$ Tanvi Lather $^{1} \cdot$ Kiran Bala $^{2} \cdot$ Jaya Shankar Kaushik $^{1}$ (D)
}

Received: 5 January 2019 / Accepted: 13 February 2019 /Published online: 7 March 2019

(C) Dr. K C Chaudhuri Foundation 2019

To the Editor: Visual evoked potential (VEP) and brainstem auditory evoked potential (BAEP) have been used to study the effects of antiepileptic drugs (AED) on visual and auditory pathways among adults, with limited studies on children [1-4]. This cross-sectional study included 36 children (aged 5-15 y) with epilepsy on AED for a duration, not less than 6 mo and 34 healthy controls. Antiepileptic drugs that were used included valproate (VPA) [14 (39\%)], phenytoin (PHT) [11(31\%)], and levetiracetam (LEV) (3(8\%)]. Rest were on combination of VPA/ clobazam (CLB) [4 (11\%)], VPA/LEV [2 (5.5\%)] and VPA/LEV/CLB [2 (5.5\%)]. Majority of the epilepsy was idiopathic [29 (81\%)] followed by neurocysticercosis in the rest seven (19\%) children.

All children underwent flash VEP and BAEP as per the standard protocol. P-100 latency on both the sides were comparable among Children with epilepsy (CWE) and controls [right side: 99.8 (20.5) vs. 104.1 (15.7); $p=0.34$; left side: 98.2 (25.8) vs. 104.5 (15.1); $p=0.25]$. Similarly, P-100 amplitudes were comparable except for N75P100 amplitude being higher in CWE compared to controls [right side: 11.8 (6.94) vs. 8.71 (3.55); $p=0.02$; left side: 11.3 (7.08) vs. 7.33 (4.03); $p=0.04]$. Peak latencies of waves I, wave III, wave V and interpeak intervals I-III and I-V of BAEP were comparable between the two groups.

AEDs decelerate central impulses and the transmission rate by increasing the effect of Gamma-aminobutyric acid (GABA) and causing sodium-channel blockade, resulting in

Presentation at the meeting: The paper was presented by Jai Behgal at Asia Pacific Congress of Pediatrics, 27-29 ${ }^{\text {th }}$ August, Bali, Indonesia.

Jaya Shankar Kaushik

jayashankarkaushik@gmail.com

1 Department of Pediatrics, Pt B D Sharma Post Graduate Institute of Medical Sciences, Rohtak, Haryana 124001, India

2 Department of Neurology, Pt B D Sharma Post Graduate Institute of Medical Sciences, Rohtak, Haryana, India prolonged latency of P100 as well as P300 [5]. Adult studies have demonstrated prolonged wave III/ V absolute latency and I-III/I-V inter-peak latency (IPL) on BAEP and prolonged P100 latencies and decreased N75P100, P100N145 amplitudes on VEP among patients on PHT, VPA and LEV [1-4]. The present study revealed that most of the VEP and BAEP characteristics were comparable between children with epilepsy on VLP, LEV and PHT either alone or in combination when compared to healthy controls. This study with a limited sample size shows that the use of conventional antiepileptic drugs for a duration of at least 6 mo in children with epilepsy are safe on the visual and auditory system.

Acknowledgements We wish to thank help of Mrs. Sunita and Mr. Wazir in coordinating performance of visual and auditory evoked potential study.

\section{Compliance with Ethical Standards}

Conflict of Interest None.

Publisher's Note Springer Nature remains neutral with regard to jurisdictional claims in published maps and institutional affiliations.

\section{References}

1. Panjwani U, Singh SH, sel Vamurthy W, Gupta HL. Brainstem auditory evoked potentials in epileptics on different anti-epileptic drugs. Indian J Physiol Pharmacol. 1996;40:29-34.

2. Verrotti A, Trotta D, Cutarella R, Pascarella R, Morgese G, Chiarella F. Effects of antiepileptic drugs on evoked potentials in epileptic children. Pediatr Neurol. 2000;23:397-402.

3. Tumay Y, Altun Y, Ekmekci K, Ozkul Y. The effects of levetiracetam, carbamazepine, and sodium valproate on P100 and P300 in epileptic patients. Clin Neuropharmacol. 2013;36:55-8.

4. Yüksel A, Sarslan O, Devranoğlu K, et al. Effect of valproate and carbamazepine on visual evoked potentials in epileptic children. Acta Paediatr Jpn. 1995;37:358-61.

5. Geller AM, Hudnell HK, Vaughn BV, Messenheimer JA, Boyes WK. Epilepsy and medication effects on pattern visual evoked potential. Doc Ophthalmol. 2005;110:121-31. 